\title{
A Tool for Performing Link Analysis, Operational Sequence Analysis, and Workload Analysis to Support Nuclear Power Plant Control Room Modernization
}

Casey R Kovesdi, Katya L Le Blanc

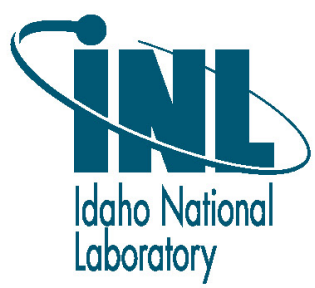




\section{DISCLAIMER}

This information was prepared as an account of work sponsored by an agency of the U.S. Government. Neither the U.S. Government nor any agency thereof, nor any of their employees, makes any warranty, expressed or implied, or assumes any legal liability or responsibility for the accuracy, completeness, or usefulness, of any information, apparatus, product, or process disclosed, or represents that its use would not infringe privately owned rights. References herein to any specific commercial product, process, or service by trade name, trade mark, manufacturer, or otherwise, does not necessarily constitute or imply its endorsement, recommendation, or favoring by the U.S. Government or any agency thereof. The views and opinions of authors expressed herein do not necessarily state or reflect those of the U.S. Government or any agency thereof. 


\section{A Tool for Performing Link Analysis, Operational Sequence Analysis, and Workload Analysis to Support Nuclear Power Plant Control Room Modernization}

Casey R Kovesdi, Katya L Le Blanc

July 2020

Idaho National Laboratory

Idaho Falls, Idaho 83415

http://www.inl.gov

Prepared for the U.S. Department of Energy Under DOE Idaho Operations Office

Contract DE-AC07-05ID14517 


\title{
A Tool for Performing Link Analysis, Operational Sequence Analysis, and Workload Analysis to Sup- port Nuclear Power Plant Control Room Moderniza- tion
}

\author{
Casey Kovesdi ${ }^{1,}{ }^{*}$, Katya Le Blanc ${ }^{1}$ \\ 1 Idaho National Laboratory, Idaho Falls, Idaho, USA \\ \{Casey.Kovesdi, Katya.LeBlanc\}@inl.gov
}

\begin{abstract}
Nuclear power continues to have an imperative role for the U.S.'s electricity generation. However, for these nuclear power plants (NPPs) to remain economically viable, new strategies for reducing operations and maintenance costs must be explored. The U.S. Department of Energy Light Water Reactor Sustainability Program is researching how to digitally transform existing analog and hybrid main control rooms into a fully integrated control room that addresses this challenge. The transformation will fundamentally change the conduct of operations for these U.S. NPPs. Human factors engineering has a vital role in this effort, where traditional task analysis methods are important in informing the design of advanced human-system interface displays. This work describes a preliminary tool to support task analysis for the development of these advanced displays. Details on the use of this tool, including the specific task analysis methods offered, are presented in this paper.
\end{abstract}

Keywords: Task Analysis · Human Factors Engineering · Nuclear Power Plant Modernization

\section{Introduction}

Nuclear power continues to have an imperative role for electricity generation in the United States (U.S.). However, for these nuclear power plants (NPPs) to remain economically viable and to support a license renewal, new strategies for reducing operations and maintenance costs must be explored. The Department of Energy (DOE) Light Water Reactor Sustainability (LWRS) Program is addressing these challenges through targeted research and development

* Corresponding author: Tel.: +1-208-526-2336 · Fax: +1-208-526-0528

E-mail address: Casey.Kovesdi@inl.gov 
$(R \& D)$ to (1) ensure existing legacy analog instrumentation and control (I\&C) systems are not life-limiting concerns for the U.S. NPP fleet, and to (2) implement advanced digital technology that enables business-driven innovation in NPP operations.

One R\&D focus is to digitally transform existing analog and hybrid main control rooms into a fully integrated control room that offers improved human-system performance and possibly reductions in required staffing. The fully integrated control room will fundamentally change the conduct of operations for the U.S. NPPs, allowing for new capabilities such as automation of manual actions, integration of plant data, and applications of advanced technologies that support decision making. For instance, rather than requiring operators to walk and visually scan the boards to find relevant information, the integrated control room will provide context-relevant information in an accessible and usable format that improves operating performance and reduces workload. Human factors engineering (HFE) has a vital role in supporting this transformation by ensuring that human-system performance is optimized, and no new human failure modes are introduced [1]. Traditional HFE methods such as task analysis are important in informing the design of new human-system interface (HSI) displays for the integrated control room such as by identifying important human actions and understanding contextual considerations that are important to HSI design [2].

This paper introduces an open-source R-based [3] tool that supports task analysis for the development of advanced HSIs like task-based displays [4] by allowing the human factors engineer to systematically map important human actions, such as specified in a procedure, to the I\&C on control boards. Collectively, the tool is intended to aid in the identification of important human actions from the task analysis outputs. This work attempts to supplement available task analysis data collection methods such as interviews, observations, verbal protocols, and walk/ talk-throughs to provide a comprehensive understanding of critical task data that supports HSI design. A brief overview of applicable task analysis techniques provided within the tool is described next.

\section{Overview of Applicable Task Analysis Techniques}

Task analysis is a methodology used to study the cognitive and physical actions needed to achieve some goal within a system [5]. For NPPs, there are complex interactions between technology, processes, and people that must be considered in task analysis. A comprehensive review of all task analysis techniques is beyond the scope of this paper. However, an overview of the tech- 
niques available within the tool is summarized next.

\subsection{Operational Sequence Diagrams}

An operational sequence is a series of control and information gathering activities completed in a specific order to accomplish a task [5, 6]. Operational sequence diagrams (OSDs) graphically represent these sequences in either temporal or spatial formats.

Temporal OSDs graphically represent the sequence of activities required by a team of agents through the course of time. Hence, one axis represents time whereas the other axis represents the different agents involved in a task [6]. An activity, such as a manual action required by an operator, is graphically presented as a single coordinate, intersecting a point in time and responsible agent (i.e., the operator). Spatial OSDs graphically represent the sequence of activities required by a team of agents in interacting within the physical environment. A diagram of a control room panel or display is typically presented where the activities are visually overlaid on the diagram to show the spatial location of where the activity takes place on the control panel or display [5]. The sequence of activities can be represented as directional arrows like the temporal OSD.

OSDs are most useful in showing the relationship of activity flow between agents through space or time. From a design standpoint, OSDs offer insights into task activities that yield the greatest benefit from redesign such as by identifying inefficient interactions between agents (e.g., such as unnecessary manual information gathering outside the control room), or unnecessary physical movements between I\&C on a control panel. The primary disadvantage of OSD is that developing the diagram can be labor intensive and take a considerable amount of time [6]. To this end, complex tasks can result in unwieldy visualizations that are difficult to interpret [5].

\subsection{Link Analysis}

Link analysis identifies the connections, or activity flow, between different parts of a system in completing a task [5]. These connections identified from link analysis are described as the links. The granularity of link analysis regarding what constitutes a part of a system is context dependent on the task analysis question at hand; however, the process of link analysis is similar. That is, sequence task data is collected like OSD, but is then aggregated across agents in a transition matrix to represent the number of times a given agent interacted with another agent. Fig. 1 illustrates an example transition matrix used in link analysis. In this example, the rows denote the initiating agent (i) whereas 
the columns denote the receiving agent (j). For example, if Agent 1 (e.g., a reactor operator) is required to monitor Agent 2 (e.g., pressurizer level) six times throughout the course of a task, the expression in the matrix would be: $L_{1,2}=6$.

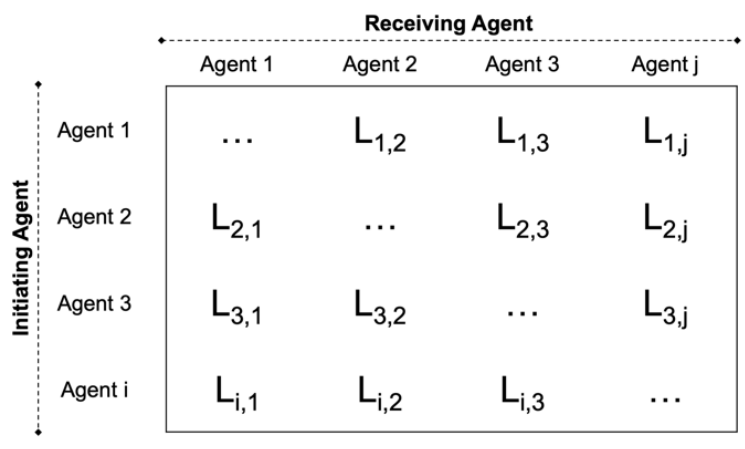

Fig. 1. Example transition matrix for link analysis.

The main output of link analysis is a visualization that shows the connections between the agents of a system to complete a task. Elements of graph theory can be used to describe these inter-relations between agents [7]. Within this context, agents are represented as nodes, whereas connections are represented as edges on the graph. The weight, or thickness, of the edge can graphically represent the frequency of connections between a pair of agents. Furthermore, extensions from graph theory and social network analysis such as the measurement of centrality may be adopted to support interpretation of link analysis. Centrality can be regarded as degree of prominence of the agents within a system [8]. An agent with the most connections between other agents has the highest centrality.

From a design standpoint, the concept of centrality can be regarded as a measure for quantifying proximity compatibility among design elements needed to complete a specific task [8]. Common centrality measures include degree, betweenness, and closeness [9]. Degree centrality can be defined as the extent to which an agent is interwoven between other agents. Betweenness centrality is the degree to which an agent acts as a mediator between the communication of two other agents. Finally, closeness centrality is the extent to which an agent utilizes the minimum number of agents between itself and each other agent [10]. 


\subsection{Timeline and Workload Profile Analysis}

Put simply, a timeline is a two-dimensional line or bar chart that graphs some set of variables across time, represented on one of the axes [5]. A Gantt chart is a commonly used timeline. For task analysis, timelines can be used for many functions, including the evaluation of workload throughout the course of time for a given task [5]. Workload profile analysis (WPA) is one such method that can be used to evaluate workload across a task [6]. With WPA, the human factors engineer in collaboration with a subject matter expert (SME) typically rates workload for each task using some subjective scaling technique [5].

The instantaneous self-assessment (ISA) technique is one such workload rating technique that collects workload at different points in time. The ISA requires the SME to rate the level of workload using a rating scale (e.g., $1=$ low; 5 = high) [6]. An advantage of ISA lies in its low-cost nature and simplicity as a single item question, which provides a quantitative estimate of workload. Due to ISA's subjective nature, an obvious pitfall is that the validity and reliability of the workload estimates are limited to the experience of the rater (i.e., the SME).

\section{A Preliminary Tool for Integrated Task Analysis}

This work presents the R-based Integrated Task Analysis Tool (R-ITAT). R-ITAT is intended to support the design of advanced HSI displays for the existing U.S. NPP fleet by providing output from OSDs, link analysis, and workload estimates at specific human actions, such as defined in a procedure, through a simple interface. R-ITAT allows a user to map important human actions described in a procedure to their spatial coordinates on a control board and temporal sequence within the task, as well as enter relevant data like WPA through a single workflow. Fig. 2 outlines the general workflow of R-ITAT. The following sub-sections describe the general workflow of using R-ITAT.

\subsection{Entering Task Analysis Data}

R-ITAT enables the user to map human actions in a procedure to a reference image through a graphical user interface. The reference image should refer to a diagram of the control panels, workstation, or individual HSI display. Fig. 2A shows an entire control panel loaded in R-ITAT as the reference image. The user points and clicks over the reference image to map each procedural step to the specific location on the image that corresponds to the step. 


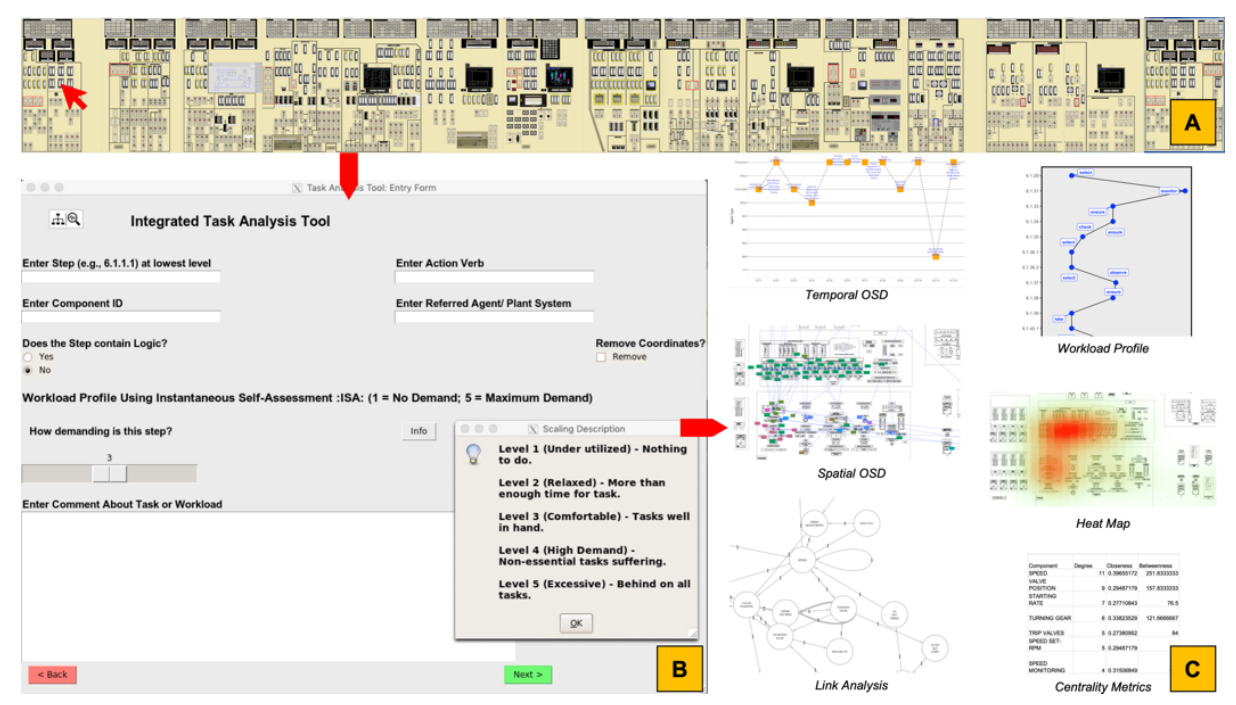

Fig. 2. General workflow of R-ITAT.

R-ITAT allows the user to enter task-relevant data in a form (Fig. 2B), including procedure step number, the action verb, referred instrumentation in the step, and corresponding agent or plant system. The WPA is completed using a scale widget that provides the ISA technique with a measurement scale analogous to that described in Stanton and colleagues [6]. There is a legend for the ISA rating scheme, provided when selecting the 'Info' button. The ISA ratings can be completed in real-time or entered in R-ITAT retrospectively. Spatial coordinates and temporal sequences are collected automatically by the tool.

\subsection{Data Processing, Analysis, and Output}

Data processing and analysis begins with R-ITAT creating a master dataset. RITAT creates visualizations from this master dataset, including: a spatial OSD, an attentional heatmap of the spatial OSD, a temporal OSD, a link analysis graph (including centrality metrics), and a WPA timeline (Fig. 2C). The spatial OSD helps visualize where the operator needs to interact, mapping the specific indications and controls, to complete a task. Each step is labeled by step number, action verb, and I\&C label. The color of the step is based on the type of action verb. The heatmap provides a gradient of where the most attention is spent on the reference image. The temporal OSD describes the temporal order of a task, visually correlating each procedural step's order to the agent described in the procedural step (e.g., a plant system, component, or plant 
personnel). Link analysis presents a network diagram where each node represents an instrumentation label. The edges represent transitions to these components from each step. Centrality metrics are also available for the link analysis. The link analysis may be used to support the identification of indications and controls that are frequently used in a specific sequence, which can inform grouping of indications for new task-based displays. Finally, the WPA diagram describes estimated workload levels across each procedural step. This data can help focus design where necessary to optimize workload.

\subsection{Applying R-ITAT to Support Task Analysis for Integrated NPP Operations}

The transformation of the existing U.S. NPP fleet into an integrated operations model requires a complete understanding of the interplay between the people, processes, and technology required for safe, effective, and efficient electricity generation. Fig. 3 illustrates the specific applications of each task analysis method offered by R-ITAT in this transformation.

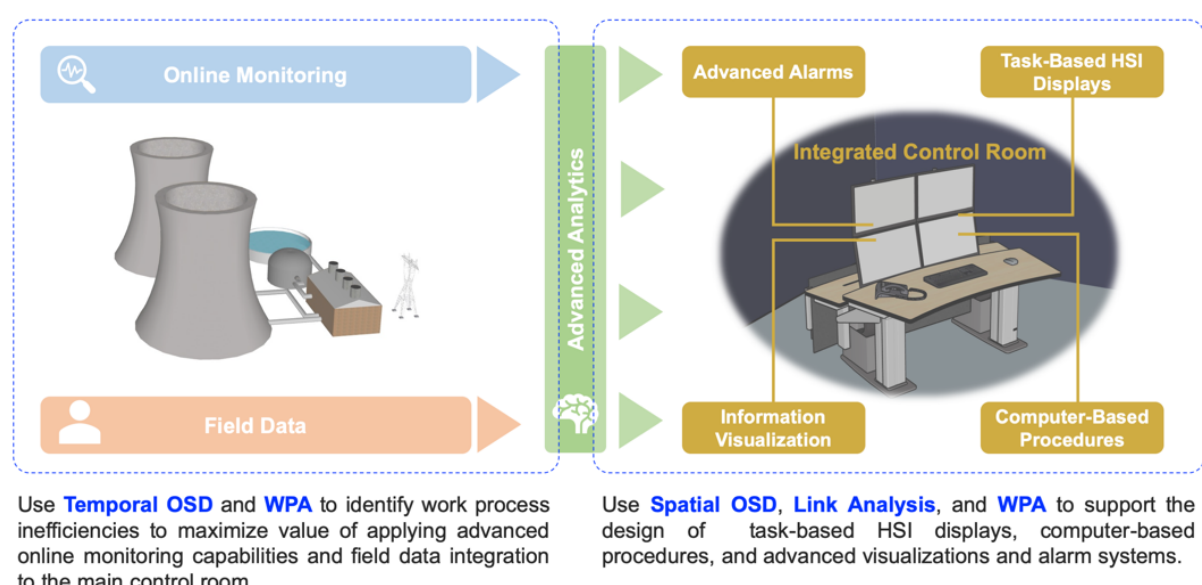

online monitoring capabilities and field data integration task-based $\mathrm{HSI}$ displays, computer-based procedures, and advanced visualizations and alarm systems.

Fig. 3. Use cases of R-ITAT in the digital transformation of U.S. NPPs as an integrated operations model.

Operational costs can be reduced by leveraging technology where there are obvious bottlenecks in the work process. For example, manual actions requiring the control room operator to contact field personnel to check the value or status of a plant component could be streamlined through the use of advanced online monitoring technology or advanced integration of field data. Temporal OSD and WPA can identify key procedural steps in these operations 
to help focus these capabilities and maximize their value. Furthermore, the application of advanced HSI displays that reduce complexity, integrate related information, and automate tedious actions can support decision-making, which may consequently support a reduced staffing footprint. Spatial OSD, link analysis, and WPA can support in developing these advanced capabilities by identifying human actions that pose greatest risk of operational difficulties and human error, resulting from an inefficient workflow, non-optimal workload, or poor grouping of information. In all cases, R-ITAT is intended to serve as additional input, as part of a larger suite of task analysis methods, to facilitate the collection of important task analysis inputs.

\section{Conclusions}

This work presents a tool to support task analysis for the development of advanced HSI displays in a fully integrated NPP control room. R-ITAT attempts to accomplish this goal by providing task analysis outputs, including temporal and spatial OSD, link analysis with measures of centrality, as well as WPA to aid in HSI design and preliminary workload assessment. Lastly, it is important to note that R-ITAT is intended to be used as one of a complete set of task analysis methods that provide a comprehensive understanding of critical task data that supports the design of advanced HSI displays.

Acknowledgments. INL is a multi-program laboratory operated by Battelle Energy Alliance LLC, for the United States Department of Energy under Contract DEAC07-05ID14517. This work of authorship was prepared as an account of work sponsored by an agency of the United States Government. Neither the United States Government, nor any agency thereof, nor any of their employees makes any warranty, express or implied, or assumes any legal liability or responsibility for the accuracy, completeness, or usefulness of any information, apparatus, product, or process disclosed, or represents that its use would not infringe privately-owned rights. The United States Government retains, and the publisher, by accepting the article for publication, acknowledges that the United States Government retains a nonexclusive, paid-up, irrevocable, world-wide license to publish or reproduce the published form of this manuscript, or allow others to do so, for United States Government purposes. The views and opinions of authors expressed herein do not necessarily state or reflect those of the United States government or any agency thereof. The INL issued document number for this paper is: INL/CON-19-56905. 


\section{References}

1. Hugo, J. V., Kovesdi, C. R., \& Joe, J. C.: The strategic value of human factors engineering in control room modernization. Progress in Nuclear Energy, 108, 381--390 (2018)

2. Joe, J., Hanes, L., \& Kovesdi, C.: Developing a human factors engineering program plan and end state vision to support full nuclear power plant modernization, INL/EXT-18-51212. Rev. 0 (2018)

3. R Core Team.: R: A language and environment for statistical computing. R Foundation for Statistical Computing, Vienna, Austria, https://www.R-project.org/ (2017)

4. Braseth, A., Nihlwing, C., Svengren, H., Veland, Ø., Hurlen, L., \& Kvalem, J.: Lessons learned from Halden project research on human system interfaces. Nuclear engineering and technology, 41(3), 215--224 (2009)

5. Kirwan, B., \& Ainsworth, L. K.: A guide to task analysis: the task analysis working group. CRC Press (1992)

6. Stanton, N. A., Salmon, P. M., Rafferty, L. A., Walker, G. H., Baber, C., \& Jenkins, D. P.: Human factors methods: a practical guide for engineering and design. CRC Press (2017)

7. Strathie, A., \& Walker, G. H.: Can link analysis be applied to identify behavioral patterns in train recorder data? Human Factors, 58(2), 205--217 (2016)

8. Dinakar, S., Tippey, K., Roady, T., Edery, J., \& Ferris, T.: Using modern social network techniques to expand link analysis in a nuclear reactor console redesign. In Proceedings of the Human Factors and Ergonomics Society Annual Meeting, 40(1), 1083-1087 (2016)

9. Freeman, L. C.: Centrality in social networks conceptual clarification. Social networks, 1(3), 215--239 (1978)

10. Guastello, S.: Human factors engineering and ergonomics: A systems approach. CRC Press (2013) 